\title{
Time from triage to initial physician assessment: A 5-year retrospective analysis
}

\author{
Mor Saban, ${ }^{1,2}$ Nadav Armoni, ${ }^{3}$ Heli Patito, ${ }^{2}$ Tal Shachar, ${ }^{4}$ Aziz Darawsha, ${ }^{2}$ Ari Lipsky \\ ${ }^{1}$ Department of Nursing, Faculty of Health and Welfare Sciences, University of Haifa, Haifa; ${ }^{2}$ Department \\ of Emergency Medicine, Rambam Health Care Campus, Haifa; ${ }^{3}$ Department of Emergency Medicine, \\ Galilee Medical Center, Nahariya; ${ }^{4}$ Azrieli Faculty of Medicine, Bar-Ilan University, Safed, Israel
}

\begin{abstract}
The aim of the study is to examine whether physicians adhere to the urgency classification as determined by the Canadian Triage and Acuity Scale. A retrospective-archive study was conducted in a tertiary hospital from January 2011 to December 2015. For each patient, we examined the relation between the urgency rating set by the triage nurse and the waiting time for the physician. Additionally, we explored the relationships between waiting times for physicians and several subgroups: patient arrival time, season of the year, assigned care area, and first consultant to examine the patient, using Analysis of Variance (ANOVA) analysis. There were 392,687 unique visits during the study period. The distribution of the classification was heterogeneous: 7,133 (1.8\%) patients were classified as Priority (P) P1; 17,318 as P2 (4.4\%); 148,657 as P3 (37.8\%); 113,502 as P4 (28.9\%); and 106,077 as P5 (27\%). Median and interquartile ranges for time from triage until physician assessment, by triage group, were: P1, 0.7 minutes (0.2-24);
\end{abstract}

Correspondence: Mor Saban, Department of Nursing, Faculty of Health and Welfare Sciences, University of Haifa, Haifa 3498838, Israel. Tel. +972.502030191.

E-mail: morsab1608@gmail.com

Acknowledgements: the authors would like to thank Ms. Kristen EbertWagner for the English editing of the manuscript.

Key words: Acuity scale; Emergency Department; Physician adherence; Triage classification.

Contributions: all authors interpreted the data and edited and approved the final article. MS, NA, HP, TS and AL drafted and conceived the study. MS, NA, AD, and AL analyzed the data, designed the study and performed data collection. MS takes responsibility for the paper as a whole. All authors attest to meeting the ICMJE.org authorship criteria.

Conflict of interest: the authors declare no potential conflict of interest.

Funding: none.

Received for publication: 26 May 2019.

Revision received: 21 August 2019.

Accepted for publication: 22 August 2019.

This work is licensed under a Creative Commons Attribution 4.0 License (by-nc 4.0).

(C) Copyright: the Author(s), 2019

Licensee PAGEPress, Italy

Emergency Care Journal 2019; 15:8307

doi:10.4081/ecj.2019.8307
P2, 35 minutes (13-76); P3, 44 minutes (21-88); P4, 45 minutes (20-87); and P5, 46 minutes (22-88). Percentages of visits that met the evaluation time goals, by triage classification, were: P1, 61\%; $\mathrm{P} 2,27 \%$; P3, 37\%; P4, 61\%; and P5, 85\%. ANOVA test for the four subgroups revealed statistically significant differences $(\mathrm{P}<.001)$. In conclusion, the standard goals for time to physician evaluation are not being met, and there is little difference in time to evaluation between the P3, P4, and P5 classifications. Initiation of system-wide changes in physician workflow and awareness may improve physician adherence to triage classification, shorten time lags, and improve patient evaluation. Further research may allow for better understanding of the factors influencing triage adherence and reinforce teamwork among Emergency Department triage nurses and physicians.

\section{Introduction}

Triage - initial assessment of patients who present to the Emergency Department (ED) - is aimed at diagnosing and prioritizing as quickly and accurately as possible the current state of the patient and determining his/her trajectory of care. ${ }^{1-4}$ To this aim, the triage nurse uses a semi-structured scoring system that categorizes the level of clinical urgency of a presenting problem, based on inputs from several sources such as the patient's subjective complaint, his or her medical background, and initial assessment. ${ }^{5,6}$

Research in ED triage has focused on the development of reliable and valid triage scales and the examination of skills of registered nurses in allocating acuity ratings. ${ }^{7-10}$ Several five-level triage scales for the ED have been developed in Anglophone countries; of these, the Canadian Triage and Acuity Scale (CTAS) is among the most commonly used. ${ }^{8}$ According to the CTAS, patients are assigned a triage level ranging from Priority $(\mathrm{P}) 1$ to Priority 5: P1 means resuscitation is needed, P2 means emergent treatment is needed, P3 means urgent treatment is needed, P4 means nonurgent treatment is needed, and P5 denotes a non-urgent visit. Patients categorized as $\mathrm{P} 1$ require immediate assessment; patients categorized as $\mathrm{P} 2, \mathrm{P} 3, \mathrm{P} 4$, or $\mathrm{P} 5$ are expected to receive physician assessment within $15,30,60$, and 120 minutes, respectively. ${ }^{10}$

In recent decades, EDs have become an especially challenging environment for making consistently accurate and timely diagnoses. ${ }^{11-14}$ Triage, consultations, admissions, discharge, and other steps in emergency care are operationally complex and usually executed under tight time constraints. Along with emergency physicians, in many countries physician consultants are an integral part of the ED staff and participate in assessing, treating, and admitting patients. ${ }^{9,10,15-19}$ These consultants include mainly general internists, surgeons, and orthopedic surgeons, who cover most ED visits. ${ }^{10}$ Additional specialties may also be present, such as neurologists, psychiatrists, and urologists. A systematic review found that 
specialty consultation was obtained for $20 \%$ to $40 \%$ of ED patients. ${ }^{19}$

In studies on ED triage classification there is much work on the effect of different variables on acuity rating, such as workload and shiftwork. ${ }^{8,14}$ High ED workload, especially overcrowding, has been shown to cause errors in triage classification. ${ }^{12-17}$ Few studies, however, have examined the relationship between P-scale classification and time to physician evaluation. ${ }^{9-11}$ Moreover, little research has examined explanatory factors that may influence the relationship between triage classification and time to physician examination.

The aim of the study is to examine whether ED physicians and consultants adhere to triage target times for initiating patient care, according to the level of urgency as defined in CTAS. Secondarily, we examined the relationships between triage-to-physician-assessment time and patient arrival time, season, assigned care level, and specialty of the first treating physician.

\section{Materials and Methods}

\section{Study design and setting}

A retrospective-archive study was conducted in the ED at Rambam Health Care Campus, a tertiary hospital that serves northern Israel, from January 2011 to December 2015. The 102-bed ED receives about 100,000 patients over 18 years old annually. Each patient who arrives at the ED is evaluated by an emergency nurse triage and referred for evaluation by one or more specialist physicians according to the nurse's impression of the relevant medical issues.

In our facility, a triage nurse is an experienced registered nurse capable of demonstrating clinical expertise in emergency settings. There are three threshold conditions for triage nurse: at least two years of experience in the current ED, an advanced course in emergency medicine (1.5 years; 434 hours of theoretical and practical studies), and a designated triage education course to prepare them for the complexities of their role (6 months).

Three specialties account for most patient referrals: internal medicine, surgery, and orthopedics. Direct referral to other specialists such as neurologists and urologists is less common. Physicians caring for patients in the ED are generally residents supervised by their respective departments. During the years this study was performed, a senior physician was in the ED providing direct overall supervision during morning and evening shifts, and was on-call during the night shift.

Upon patient arrival, the triage nurse assigns the triage P-scale to the patient and directs the patient to a particular treatment area in the ED. These areas include the resuscitation bay, generally for $\mathrm{P} 1$ and $\mathrm{P} 2$ patients, which has 6 beds; the emergent care area, for P2 through P4 patients, with 96 beds and the urgent care area, for P4 and P5 patients, where chairs are available while waiting evaluation in a consultation room. Each wing is managed by a physician, two nurses and a technician (for performing ECGs, starting intravenous lines, and drawing laboratory tests) each shift. On average, 310 patients arrive daily, with approximately 150 patients cared for in the emergent care area, 150 in the urgent care area, and 10 in the resuscitation bay.

\section{Methods and measurements}

Data were collected retrospectively from electronic medical records maintained by the hospital.

We collected and measured the following variables for each patient: arrival time at the ED, which we further categorized into three main shifts: morning (7:00-15:00), evening (15:00-23:00), and night (23:00-7:00); initial assigned care area (resuscitation bay, emergent care area, or urgent care area); and ED process and outcome variables including time of triage, P-scale classification, time of referral to specialist physician, physician specialty, time to physician evaluation, time to final decision, disposition decision (discharge/hospitalization), and in-hospital mortality. Time to physician evaluation was determined by noting the first time the physician documented in the medical record or placed an order (usually for a laboratory, medication, or imaging study). We included only records that contained information about triage level and where time from triage to physician evaluation could be calculated. For patients who were referred to several specialists, we considered only the time of the initial evaluation.

We compared the triage category set by the triage nurse and the time to initial physician evaluation. We used the accepted CTAS goals and published recommendations for adherence percentages as the gold standards: ${ }^{20}$ patients categorized as $\mathrm{P} 1$ require immediate physician evaluation ( $<5 \mathrm{~min}$.), with $98 \%$ achieving this goal; patients categorized as $\mathrm{P} 2, \mathrm{P} 3, \mathrm{P} 4$, and $\mathrm{P} 5$ should receive physician evaluation within $15,30,60$, and 120 minutes, respectively, with $95 \%, 90 \%, 85 \%$, and $80 \%$, respectively, achieving these goals for time to evaluation. Additionally, we assessed several subgroups for trends in evaluation time: patient arrival time (morning/evening vs night shift), season (winter/other), area where patient was examined (resuscitation bay, emergent care area, or walk-in clinic), and first physician specialty to examine the patient (internist, surgeon, or orthopedist). To help assess our triage validity, we compared the triage assessment with in-hospital mortality and hospital length of stay. ${ }^{20}$

\section{Data management and statistical analysis}

Statistical analyses were performed using descriptive data analysis, including ranges, means, medians, standard deviations, and interquartile ranges (IQRs) for continuous variables and frequencies and percentages for categorical variables. ANOVA test was performed for several subgroups: patient arrival time, season, assigned care area, and first consultant to examine the patient. The level of significance for all statistical analyses was $5 \%$. Data analysis was performed using R, version 3.2.4 (R Core Team, Vienna, Austria). Data anonymization was achieved at the level of our bioinformatics department: no patient identifying data were received for this analysis, including name, birthdate, gender, or national identification number.

This study was approved by the institutional Helsinki committee (0577-15-RMB).

\section{Results}

Of the 616,197 ED patient records from the study period, $50,610(8.3 \%)$ were excluded because there was no recorded triage level; $8,103(1.3 \%)$ were excluded because they were missing either the triage time or the physician evaluation time or both; and $164,797(26.7 \%)$ records were removed because they were duplicates (i.e., physician specialist involvement subsequent to the first physician evaluation). The remaining 392,687 unique visits were included in the analysis (Figure 1).

The number of ED visits grew significantly at a rate of $5 \%$ per year over the study period, from 68,197 in 2011 to 86,997 in 2015 $(\mathrm{P}<.001)$. Through this period, there was an overall increase in $\mathrm{P} 1$, $\mathrm{P} 3$, and $\mathrm{P} 4$ classifications (by 10\%, 18\%, and 13\%, respectively), 
with a concomitant reduction in P2 and P5 classifications (by 3\% and $6 \%$, respectively). Overall, the mean patient age was $67 \pm 20$ years; the average age increased by 3.7 years over the study period. Fifty-two percent of the patients were male, which was quite stable over the study period. Table 1 displays time to evaluation by triage category. The highest adherence to the goals for time to physician assessment was at P5, where $85 \%$ met the target of 120 minutes; the CTAS recommendation is $80 \%$. Adherence for $\mathrm{P} 1$ and $\mathrm{P} 4$ was fair, with $61 \%$ of the cases meeting the targets, whereas adherence for $\mathrm{P} 2$ and $\mathrm{P} 3$ was poor, with $27 \%$ and $37 \%$, respectively, meeting the time-to-evaluation targets. No substantial differences were found between the mean and median waiting times for P2, P3, P4, and P5. The times to evaluation among the triage levels were statistically different $(\mathrm{P}<0.001)$. With regard to the potential factors that affect physician adherence, although more patients $(43.5 \%)$ arrived during morning shifts, time to physician evaluation was slightly shorter during morning vs non-morning (evening and night) shifts (Table 2). These differences were found to be statistically significant $(\mathrm{P}<.001)$. Patients were evaluated slightly faster in winter. Nevertheless, the above results for both morning shift and winter season are quite far from the CTAS targets. As far as exam-

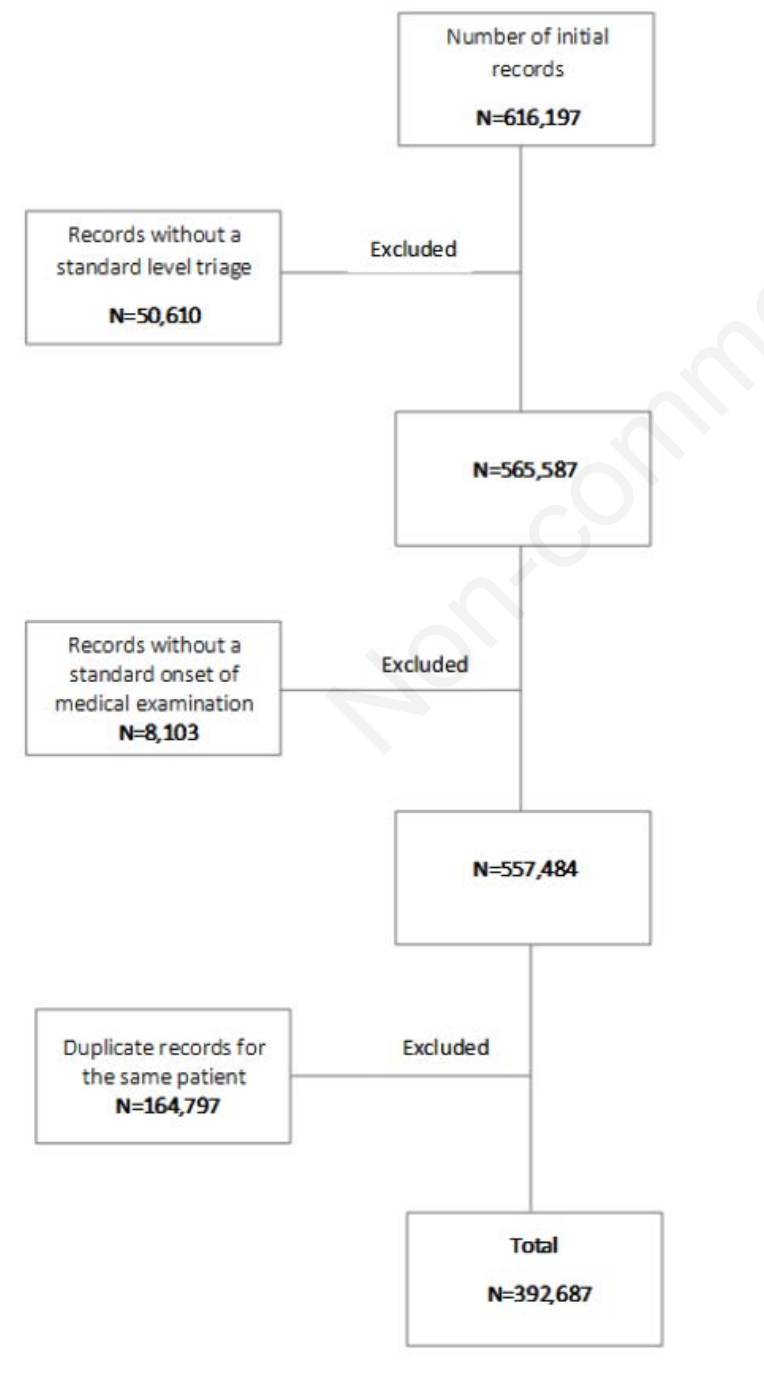

Figure 1. Research flow diagram. ination area, patients admitted to the resuscitation bay were rapidly evaluated (median time 2.2; IQR 0.2-33.4). For emergent and urgent care areas, evaluation times were similar (median time and IQR for emergent care area, 44.8 [20.0-91.9]; for urgent care, 46.1 [21.8-87.2]). Seventy-five percent of patients seen in the ED were first evaluated by one of the following specialists: internist $(41.8 \%)$, surgeon $(14.5 \%)$, and orthopedist $(19.1 \%)$. With regard to wait times, surgeons met the CTAS targets better than the two other specialists in levels P2 through P5 (Table 2).

Time to physician evaluation differed significantly by each of patient arrival time, season of the year, assigned care area, and first consultant specialty $(\mathrm{P}<0.001)$. Figure 2 depicts changes in median time to medical examination, by priority level, during the study period. For P1, time to medical examination improved from 2011 to 2012 (median: $10.6 \mathrm{~min}$. vs $0.5 \mathrm{~min}$.) and was sustained thereafter; for P2 there was no substantial change; and time to medical examination worsened for patients in $\mathrm{P} 3$ (37.5 min. vs $45.3 \mathrm{~min}$.), P4 (39.3 min. vs $50.1 \mathrm{~min}$.), and P5 (41.1 min. vs $53.6 \mathrm{~min}$. .). Adherence to the triage goals improved from 2011 to 2015 for P1 (44\% vs 65\%), were fairly stable for P2 (28\% vs 27\%), and worsened for P3 through P5 (Figure 3). Forty-two percent of the patients who visited the ED were hospitalized during the study

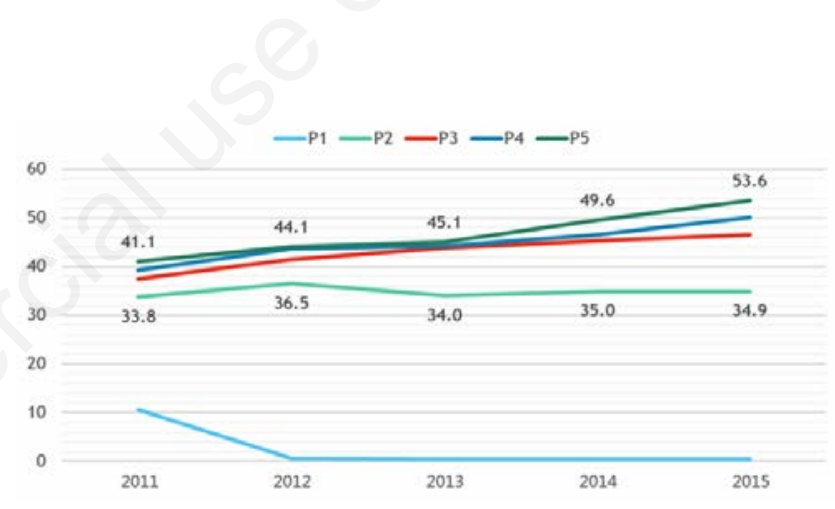

Figure 2. Median time (minutes) to physician evaluation, by Canadian Triage and Acuity Scale P-level, 2011-2015. P1, resuscitation; P2, emergency treatment; P3, urgent treatment; P4, non-urgent treatment; $P 5$, non-urgent visit.

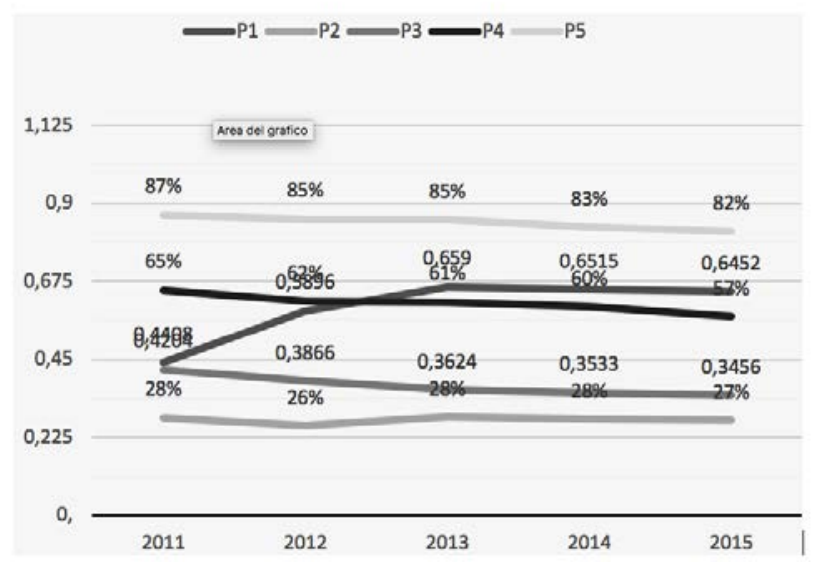

Figure 3. Percentage of triage goals met during the study period, by Canadian Triage and Acuity Scale P-level, 2011-2015. P1, resuscitation; P2, emergency treatment; P3, urgent treatment; P4, non-urgent treatment; P5, non-urgent visit. 
period. Figure 4 illustrates the relationship between P-scale classification and hospitalization/in-hospital mortality. A positive association emerged between higher priority classification and both hospitalization and in-hospital mortality. ${ }^{20}$

\section{Discussion}

Effective triage prioritizes patients who require quick and intensive care..$^{21,22}$ Adherence to triage target times is essential to providing rapid and appropriate treatment according to patient condition. ${ }^{8,21}$ However, in our study the standard goals for time to evaluation were not being met; only $54 \%$ of patients were evaluated within the recommended window.

The percentage of patients in priority levels P1, P4, and P5 who were evaluated within the recommended time was reasonable, although not ideal. What stands out, however, is that for patients in levels P2 through P5, there is little variation in time to physician evaluation. This finding, unlike those of previous studies, , 3,4,7,22 suggests that our patients are being evaluated based on their presentation time irrespective of their triage priority level. Additionally, it suggests that the ED is understaffed by physicians: if we were to shift physician attention to higher-priority-level patients, time to evaluation for lower-priority-level patients, which currently has significant room for improvement, would presumably worsen.

The hospitalization rate $(42 \%)$ remained stable throughout the study period. Alongside this, one must consider that each year there was a $5.5 \%$ increase in the number of patients admitted to the ED; thus, each year more and more patients were hospitalized, and the organizational load increased. EDs are usually busy places

Table 1. Descriptive statistics for the study sample $(\mathrm{N}=392,687)$.

\begin{tabular}{lcccccc}
$\begin{array}{l}\text { Urgency } \\
\text { scale }\end{array}$ & $\begin{array}{c}\text { Desired time } \\
\text { to medical examination } \\
\text { by CIAS, in minutes }\end{array}$ & $\begin{array}{c}\text { Mean time to } \\
\text { examination, } \\
\text { in minutes }\end{array}$ & $\begin{array}{c}\text { Median (IOR) time } \\
\text { to examination, } \\
\text { in minutes }\end{array}$ & $\begin{array}{c}\text { CTAS } \\
\text { recommendation } \\
(\%)\end{array}$ & $\begin{array}{c}\text { Percentage of } \\
\text { cases meeting } \\
\text { target }\end{array}$ & $\begin{array}{c}\text { Number of ED } \\
\text { visits } \\
\text { (\% total) }\end{array}$ \\
P1 & Immediate & 25.3 & $0.7(0.7-24.3)$ & 98 & 61 & $7,133(1.8)$ \\
P2 & 15 & 60.8 & $35.1(13.1-75.9)$ & 95 & 27 & $17,318(4.4)$ \\
\hline P3 & 30 & 69.5 & $43.5(19.8-87.3)$ & 90 & 37 & $148,657(37.8)$ \\
P4 & 60 & 68.3 & $45.3(21.1-88.4)$ & 85 & 61 & $113,502(28.9)$ \\
\hline P5 & 120 & 66.3 & $46(21.5-87.8)$ & 80 & 85 & $106,077(27)$ \\
\hline
\end{tabular}

CTAS, Canadian Triage and Acuity Scale; IQR, interquartile range; ED, emergency department; P1, resuscitation; P2, emergency treatment; P3, urgent treatment; P4, non-urgent treatment; P5, non-urgent visit.

Table 2. Descriptive statistics for the study sample, by patient arrival time (shift), season, patient examination site, and specialty of the first evaluating physician $(\mathrm{N}=392,687)$.

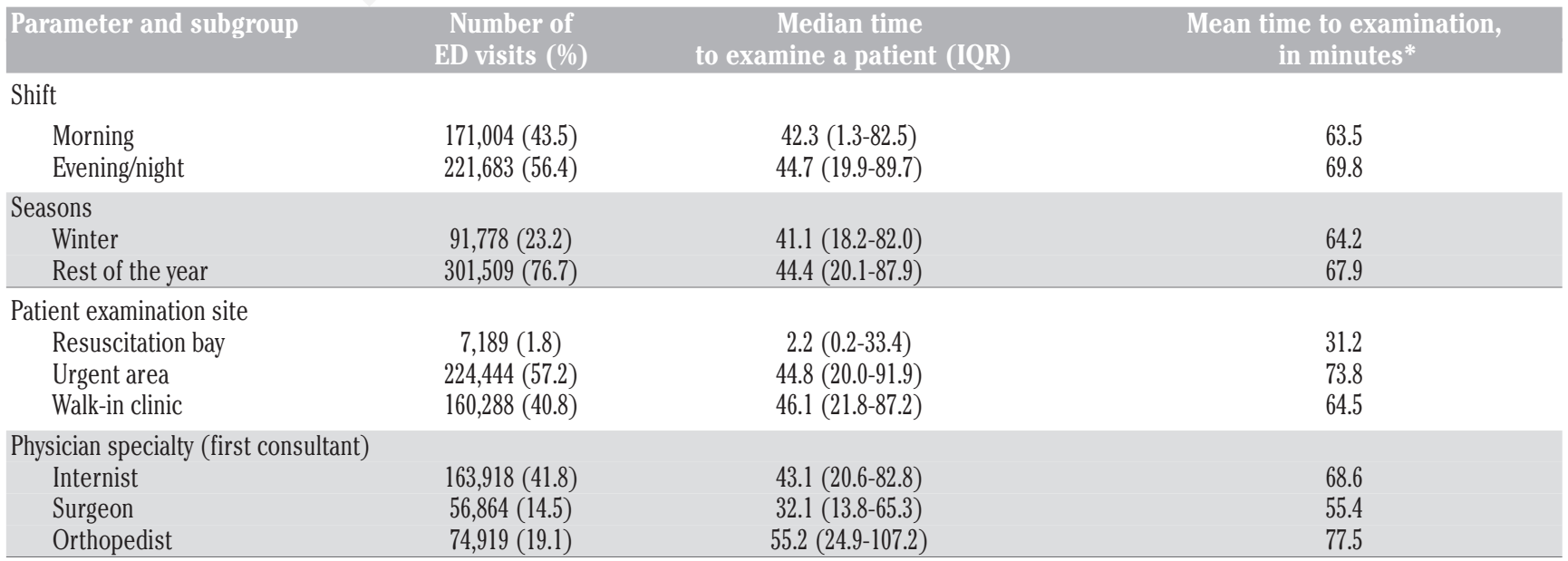

ED, emergency department; IQR, interquartile range. *Within each parameter, the distribution of mean times to examination was significantly different $(\mathrm{P}<0.001)$ across the subgroups. 
where physicians and staff might lack time to perform optimal care and surveillance due to overcrowding, and where there is little continuity of care. ${ }^{23}$ The simultaneous management of multiple patients and the need for quick decision making with incomplete information can render the ED chaotic. ${ }^{24}$ Thus, guidelines and protocols have become an important aspect of emergency care clinical practice. ${ }^{25}$ A guideline consists of systematically developed recommendations to assist practitioners and patient decisions about appropriate health care for specific clinical circumstances. ${ }^{26}$ Despite the existence of guidelines and protocols, a gap between recommended care and clinical practice often exists, as we demonstrate in the current study vis-à-vis triage.

With regard to possible factors affecting time to evaluation, physicians were better at meeting the CTAS goals during morning rather than during evening and night shifts. This could be because more medical staff are present during morning shifts. ${ }^{27}$ The second factor, season, was not found to be related to physician adherence. The results for all four factors considered here are statistically significant because of the large numbers of subjects, so we it is important to consider clinical relevance. For season, we do not believe the 2.3-minute difference between winter and not-winter to be clinically relevant. In addition, we did not find noteworthy differences between the number of ED admissions in the winter as compared with the other seasons. This finding contradicts previous studies that found winter to be the busiest season in the $\mathrm{ED}^{28}$ and that both ED occupancy and mortality rates increase during winter. ${ }^{29}$ However, a study of the relationship between ED overcrowding and mortality showed that testing for winter seasonal confounding revealed no significant effect. ${ }^{30}$ Among the evaluation areas, the urgent care area had the longest time to examination. In this site patients are examined by diverse specialist physicians, which may explain the longer wait times. ${ }^{13,31}$ Further, among the core ED specialties, time to orthopedic examination was longest. This finding contrasts with a study that found that time to orthopedist consultation is one of the shortest in the ED. ${ }^{10}$ These discrepancies are very likely site-specific, with local hospital flow, workforce, and population factors contributing.

Our results raise questions about how the ED queue is managed by physicians. It is unclear whether this is part of applying the first in, first out principle or whether queuing management follows other parameters that are not included in the CTAS, such as physician availability. We note that our triage seems appropriate overall, given the graded increases in admission rates and mortality, by priority level.

The study showed noticeable heterogeneity between triage nurse classification and the time to physician assessment. This diversity suggests that patient's safety during triage may depend to some extent on the ED team. Implementation of educational processes may minimize the gap between recommended care and clinical practice. First, a theoretical learning process, including designated staff meetings discussing triage scales and the importance of collaboration between triage nurses and physicians, may increase awareness and contribute to increased responsiveness of the physician to the triage classification set by the triage nurse. This would emphasize the importance of knowledge acquisition for improving triage decision. Second would be the use of a practical learning process using patient simulations based on authentic patient situations from the clinical ED experience; this would help simulate practical decision-making. Following the identification of variations of thinking strategies, a productive discussion can improve staff approach for future patients. Consequentially, the examination of physician adherence and collaboration with triage nurses, will allow for repetitive evaluation of misclassified cases.

\section{Limitations}

This study has several limitations. First, the results for the P1 priority level should be considered carefully. P1 patients may very often receive quick and proper treatment in the resuscitation bay, and only after they are stabilized do medical staff document their medical records. The difficulties of documenting accurate activity of severe patients in the ED are well known..$^{32}$ This phenomenon likely also impacts the P2-P5 results, though our experience is that the effect is much more attenuated in these groups.

Second, a high percentage of medical records lacked information about standard-level triage or standard onset of medical examination. This was likely due to problems in the evolving electronic medical record.

Third, the results are based on CTAS triage goals for determining a patient's urgency level; using other tools might have yielded different results. In addition, it is unclear whether measuring adherence to triage targets best reflects physician performance. Fourth, as mentioned earlier, although statistical significance was found in all groups, it is likely due to the large sample size and may not reflect clinical relevance. Finally, this study suffers from all the usual biases inherent in a retrospective chart study. ${ }^{33}$

\section{Conclusions}

Our institution does not meet CTAS recommended targets for triage to provider evaluation time. The primary reason seems to be that physicians are evaluating patients based on waiting time, without regard for their triage classification. Physician understaffing may also play a significant role. Training programs should be developed and constructed to improve physicians' responsiveness and awareness, and physician staffing should be re-evaluated.

\section{Conclusions}

1. Beardsell I, Robinson S. Can emergency department nurses performing triage predict the need for admission? Emerg Med J 2011;28:959-62.

2. Göransson KE, Ehrenberg A, Marklund B, Ehnfors M. Emergency department triage: Is there a link between nurses' personal characteristics and accuracy in triage decisions? Accid Emerg Nurs 2006;14:83-8.

3. Hunold KM, Pereira GF, Jones CW, et al. Priorities of care among older adults in the emergency department: a cross-sectional study. Acad Emerg Med 2016;23:362-5.

4. Considine J, LeVasseur SA, Villanueva E. The Australasian Triage Scale: Examining emergency department nurses' performance using computer and paper scenarios. Ann Emerg Med 2004;44:516-23.

5. Cameron A, Ireland AJ, McKay GA, et al. Predicting admission at triage: are nurses better than a simple objective score? Emerg Med J 2017;34:2-7.

6. Roscoe LA, Eisenberg EM, Forde C. The role of patients' stories in emergency medicine triage. Health Commun 2016;31:1155-64.

7. Fernandes CMB, Tanabe P, Gilboy N, et al. Five-level triage: a report from the ACEP/ENA Five-level Triage Task Force. J Emerg Nurs 2005;31:39-50.

8. Goransson K, Ehrenberg A, Marklund B, Ehnfors M. Accuracy and concordance of nurses in emergency department triage. Scand J Caring Sci 2005;19:432-8. 
9. Brick C, Lowes J, Lovstrom L, et al. The impact of consultation on length of stay in tertiary care emergency departments. Emerg Med J 2014;31:134-8.

10. Yoon P, Steiner I, Reinhardt G. Analysis of factors influencing length of stay in the emergency department. CJEM 2003;5:155-61.

11. van der Veen D, Heringhaus C, de Groot B. Appropriateness, reasons and independent predictors of consultations in the emergency department (ED) of a Dutch Tertiary care center: a prospective cohort study. PLoS One 2016;11:e0149079.

12. Kelen GD, Scheulen JJ, Hill PM. Effect of an emergency department (ED) managed acute care unit on ED overcrowding and emergency medical services diversion. Acad Emerg Med 2001;8:1095-100.

13. Marmor YN, Golany B, Israelit S, Mandelbaum A. Designing patient flow in emergency departments. IIE Trans Healthc Syst Eng 2012;2:233-47.

14. van der Linden MC, Meester BEAM, van der Linden N. Emergency department crowding affects triage processes. Int Emerg Nurs 2016;29:27-31.

15. Adriaenssens J, De Gucht V, Van Der Doef M, Maes S. Exploring the burden of emergency care: predictors of stresshealth outcomes in emergency nurses. J Adv Nurs 2011;67:1317-28.

16. Göransson KE, Ehnfors M, Fonteyn ME, Ehrenberg A. Thinking strategies used by Registered Nurses during emergency department triage. J Adv Nurs 2008;61:163-72.

17. Forsgren S, Forsman B, Carlström ED. Working with Manchester triage - Job satisfaction in nursing. Int Emerg Nurs 2009; 17:226-32.

18. Woods RA, Lee R, Ospina MB, et al. Consultation outcomes in the emergency department: exploring rates and complexity. CJEM 2008;10:25-31.

19. Lee RS, Woods R, Bullard M, et al. Consultations in the emergency department: a systematic review of the literature. Emerg Med J 2008;25:4-9.

20. Kayden S, Anderson PD, Freitas R, Platz E, eds. Emergency department leadership and management. Cambridge, UK: Cambridge University Press; 2014.

21. Murray M, Bullard M, Grafstein E, et al. Revisions to the Canadian Emergency Department Triage and Acuity Scale Implementation Guidelines. CJEM 2004;6:421-7.

22. Vlahaki D, Milne WK. Meeting Canadian emergency depart- ment triage and acuity scale benchmarks in a rural emergency department. Can J Rural Med 2009;14:101-4.

23. Cowan RM, Trzeciak S. Clinical review: Emergency department overcrowding and the potential impact on the critically ill. Crit Care 2005;9:291.

24. Freund Y, Goulet H, Leblanc J, et al. Effect of systematic physician cross-checking on reducing adverse events in the emergency department. JAMA Intern Med 2018;178:812.

25. Ebben RH, Vloet LC, Verhofstad MH, et al. Adherence to guidelines and protocols in the prehospital and emergency care setting: a systematic review. Scand J Trauma Resusc Emerg Med 2013;21:9.

26. Lohr K, Field M, eds. Clinical practice guidelines: directions for a new program. Washington, DC: National Academies Press (US); 1990.

27. Patvivatsiri L. A simulation-based approach for optimal nurse scheduling in an emergency department. MSc Diss, Virginia Tech, Blacksburg, Virginia; 2003. Available from: https://vtechworks.lib.vt.edu/handle/10919/43867. Accessed: November 5, 2018.

28. Dong SL, Bullard MJ, Meurer DP, et al. Predictive validity of a computerized emergency triage tool. Acad Emerg Med 2007; 14:16-21.

29. Richardson DB. Increase in patient mortality at 10 days associated with emergency department overcrowding. Med J Aust 2006;184:213-6.

30. Sprivulis P, Da Silva J, Jacobs IG, et al. The association between hospital overcrowding and mortality among patients admitted via Western Australian emergency departments. Med J Aust 2006;184:208-12.

31. Northington WE, Brice JH, Zou B. Use of an emergency department by nonurgent patients. Am J Emerg Med 2005;23: 131-7.

32. Au-Yeung SWM, Harrison PG, Knottenbelt WJ. A queueing network model of patient flow in an accident and emergency department. London, UK: Imperial College London; 2006. Available from: http://www.doc.ic.ac.uk/ wjk/publications/ auyeung-harrison-knottenbelt-esm-2006.pdf. Accessed: November 5, 2018.

33. Kaji AH, Schriger D, Green S. Looking through the retrospectoscope: reducing bias in emergency medicine chart review studies. Ann Emerg Med 2014;64:292-8. 